Vitae Johannis Chrysostomi. Ed. François HaLKIN, Douze récits byzantins sur saint Jean Chrysostome. Subsidia Hagiographica 60, Bruxelles 1977.

Vitae Simeonis Stylitae (Theodoret h.r. XXVI, Antonius, syrische Lebensbeschreibung in Deutsch). Ed. Heinrich LietzmanN, Leipzig 1908. Darin die deutsche Übers. der syrischen Vita von Heinrich Hilgenfeld. Gr.-frz. Hippolyte Delehaye, Subsidia Hagiographica 14, Bruxelles 1923, engl. Übers. mit Einführung Robert DoraN, Kalamazoo, Mich. 1992, die lat. Übers. des Antonius in PL 73, 325-334.

Vita Simeonis Stylitae Iunioris. Ed. Paul VAN DEN VEN, La Vie ancienne de S. Syméon Stylite le Jeune. 2 Bde. Subsidia Hagiographica 32. Bruxelles 1962-1970.

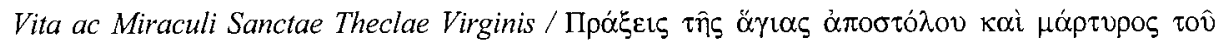

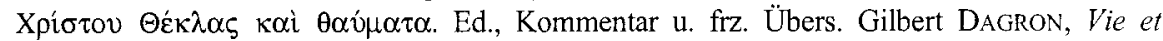
Miracles de Sainte Thècle. Subsidia Hagiographica 62. Bruxelles 1978. [Mir.]

Zacharias Rhetor, Historia Ecclesiastica. Ed. Emest W. BROOKs, CSCO 83/84, Scriptores Syri 41/42. Louvain 1924. Dt. Übers. Karl AHRENS / Heinrich KRÜGER, Scriptores sacri et profani III, Jena1899. Engl. Übers. Frederick J. HamiLton / Emest W. BroOKS, CSCO 87/88, Scriptores Syri 41/42, London 1899.

- Vita Severi. Ed. u. Syrisch-französisch Marc-Antoine KUGENER, PO II, Paris 1907.

Zosimus, Historia nova, ed. Ludwig MENDELssoHn. Leipzig 1887, Griechisch-frz. und Kommentar François Paschoud, Bd. I-IV. Paris ${ }^{2} 2000-22003$. Dt. Übers. Otto VeH, mit Kommentar von Stefan Rebenich, BGL 31. Stuttgart 1990.

\title{
2. Epigraphische, numismatische und papyrologische Quellen
}

Ägyptische Urkunden aus den Staatlichen Museen Berlin. Griechische Urkunden, XII. Band: Papyri aus Hermoupolis. Ed. Herwig MAEHLER, Berlin 1974 [BGU]

BEAN, George E. / Mitford, Terence B.: Journeys in Rough Cilicia in 1962 and 1963 (= Österreichische AdW, phil.-hist. Klasse, Denkschriften Bd. 85). Wien 1965. [BM I]

- Journeys in Rough Cilicia 1964-1968 (= Österreichische AdW, phil.-hist. Klasse, Denkschriften Bd. 102, ETAM 3). Wien 1970. [BM II]

The Roman Imperial Coinage Vol. X. The Divided Empire and the Fall of the Western Parts, AD 395-491. Ed. John P. C. KENT. London 1994. [RIC X]

Corpus Inscriptionum Latinarum Consilio et auctoritate Academiae litterarum Borussicae editum. Berlin 1869 ff. [CIL]

Dagron, Gilbert / FeIssel, Denis: Inscriptions de Cilicie, Travaux et Mémoires du centre du recherche d'histoire et civilisation de Byzance, Monographies 4. Paris 1987.

Greek Papyri in the British Museum, 3 Bde. Ed. F. G. Kenyon / H. I. Bell. London 1893-1907. [P. Lond. $]$

HaGel, Stefan / TOMASchiTz, Kurt: Repertorium der westkilikischen Inschriften, ETAM 22; Österreichische AdW, phil.-hist. Klasse, Denkschriften 265. Wien 1998. [Repertorium]

HaHN, Wolfgang: Moneta Imperii Byzantini 1, Österreichische AdW, phil.-hist. Klasse, Denkschriften, Bd. 109. Wien 1973. 
Heberdey, Rudolf / Wilhelm, Adolf: Reisen in Kilikien, Österreichische AdW, phil.-hist. Klasse, Denkschriften 44. Wien 1896.

HEITSCH, Ernst: 'Die griechischen Dichterfragmente der römischen Kaiserzeit', Abh. d. AdW Göttingen, phil.-hist. Kl. 3, 49, Göttingen 1961.

HerzFeld, Ernst / GuYer, Samuel: Meriamlik und Korykos. Zwei christliche Ruinenstätten des Rauhen Kilikiens. (= Monumenta Asiae Minoris Antiqua II). Manchester 1930. [MAMA II]

HILL, George F.: Catalogue of the Greek Coins of Lycaonia, Isauria, and Cilicia (= Catalogue of the Greek Coins in the British Museum 21). London 1900, ND Bologna 1964. [CGC]

Inscriptiones antiquae orae septentrionalis Ponti Euxini Graecae et Latinae. Ed. Vasilij V. LATYSCHEV. Petersburg 1890, ND Hildesheim 1965. [IPE]

Inscriptiones Graecae. Ed. Preuß. / Berlin-Brandenburgische AdW. Berlin 1873 ff. $[I G]$

Inscriptiones Graecae ad res Romanas pertinentes. Tomus III. Ed. René CAGNAT. Paris 1906. $[I G R]$

Inscriptiones Latini Selectae, 3 Bde. Ed. Hermann Dessau. Berlin 1892-1916, ND Berlin ${ }^{3} 1962$. $[I L S]$

Inscriptiones latinae liberae rei publicae. Ed. Attilio DEGRASSI. Göttingen 1957. [ILLRP]

The inscriptions of Central Pisidia. Including texts from Kremna, Ariassos, Keraia, Hyia, Panemoteichos, the Sanctuary of Apollo of the Perminoundeis, Sia, Kocaaliler, and the Döşeme Boğazı ( $=$ Inschriften griechischer Städte aus Kleinasien 57), hrsg. v. G. H. R. HoRSLEY u. Stephen MitChELl, Bonn 2000.

KeIL, Josef / WiLhelm, Adolf (Hrsg.): Denkmäler aus dem Rauhen Kilikien (= Monumenta Asiae Minoris Antiqua III). Manchester 1931. [MAMA III]

LAMINGER-PASCHER, Gertrud: Die kaiserzeitlichen Inschriften Lykaoniens. Faszikel 1: Der Süden (= Österreichische AdW, phil.-hist. Klasse, Denkschriften 232, ETAM 15). Wien 1992.

LancKoronski, Karl Graf: Städte Pamphyliens und Pisidiens, 2 Bde. Prag-Wien-Leipzig 1890 1892.

Notitia Dignitatum, accedunt Notitia Urbis Constantinopolitanae et Latercula Provincianum. Ed. Otto SEECK, Frankfurt a.M. 1876. [ND]

Notitiae episcopatuum Ecclesiae Constantinopolitanae. Ed. Jean DARrouzÈs (= Géographie ecclésiastique de l'empire byzantin 1). Paris 1981.

Orientis Graeci Inscriptionis Selectae. 2 Bde. Ed. Wilhelm DitTENBERgER. Leipzig 1903-1905, ND Hildesheim 1960. [OGIS]

Paribeni, Roberto / Romanelli, Pietro: 'Studi e ricerche archeologiche nell'Anatolia Meridionale' in: Monumenti Antichi 23 (1914), 5-274. [PR]

Res Gestae Divi Saporis. Ed. Philip HuYSE, Die dreisprachige Inschrift Šābuhrs I. an der Ka'ba-i Zardušt (ŠKZ), 2 Bde. (= Corpus Inscriptionum Iranicarum, Part III: Pahlavi Inscriptions), Bd. 1: Einleitung, Texte, Übersetzungen, Indizes, Glossar; Bd. 2: Kommentar, Appendix, Tafeln. London 1999. [RGDS]

Robertson, Anne S.: Roman Imperial Coins in the Hunter Coin Cabinet Vol. V: Diocletian (Reform) to Zeno. Oxford 1982.

STERrETT, John Robert Sitlington: The Wolfe Expedition to Asia Minor, Papers of the American School of Classical Studies at Athens $3(1883 / 1884)$. Boston 1888. [WE] 
Supplementum Epigraphicum Graecum. Amsterdam 1923 ff. [SEG]

Sylloge Inscriptionum Graecarum. Ed. W. DitTENBERGER. Hildesheim 1960. [SIG]

Sylloge Nummorum Graecorum. Ed. Hans v. AuLock, Berlin 1964-1966. [SNG]

Tituli Asiae Minoris II. Pamphylia. Ed. Ernst KalinKA, Wien 1944. [TAM II]

Tolstol, Jean: Monnaies Byzantines. St. Petersburg 1912-1914, ND Amsterdam 1968 (russ.).

TOMASCHITZ, Kurt: Unpublizierte Inschriften Westkilikiens aus dem Nachlaß Terence B. Mitfords (= Österreichische AdW, phil.-hist. Klasse, Denkschriften 264, ETAM 21). Wien 1998.

Wrotн, Warwick: Catalogue of the Coins of the Vandals, Ostrogoths and Lombards, and of the Empires of Thessalonica, Nicaea and Trebizond, in the British Museum. London 1911.

ZACOS, Georges / VeGLeRY, Alexander: Byzantine Lead Seals I. Basel 1972.

\section{Nachschlagewerke, Quellenkunden, Atlanten und Bibliographien}

L'Année Philologique. Bibliographie critique et analystique de l'Antiquité Gréco-Latine. Fondée par Jean Marouzeau. Paris $1928 \mathrm{ff}$.

Assemanus, Ioseph Simonius / Evodius, Stephanus: Bibliothecae Apostolicae Vaticanae codicum manuscriptorum catalogus in tres partes distributus. 1. Teil Bd. 3 Rom 1759, ND Paris 1926.

Bagnall, Roger S. / Cameron, Alan / SchwarTZ, Seth R. / Worp, Klaas A.: Consuls of the Later Roman Empire (= Philological Monographs of the American Philological Association 36). Atlanta, Georgia 1987. [CLRE].

BAUER, Walter: Griechisch-deutsches Wörterbuch zu den Schriften des Neuen Testaments und der frïhchristlichen Literatur. Berlin-New York ${ }^{6} 1988$.

Bickerman, Elias J.: Chronology of the Ancient World. London ${ }^{2} 1980$.

Degrassi, Attilio: I fasti consolari dell' Impero romano dal 30 avanti cristo al 613 dopo Cristo. Rom 1952.

Dictionnaire d'Archéologie Chrétienne et de Liturgie. Paris 1924 ff. [DACL]

Dictionnaire d'Histoire et de Géographie Ecclésiastiques. Paris 1912 ff. [DHGE]

Hammond, Nicholas G. L.: Atlas of the Greek and Roman World in Antiquity. Park Ridge, NJ 1981.

JusTI, Ferdinand: Iranisches Namenbuch. Marburg 1895.

Karayannopoulos, Johannes / WeISS, Günther: Quellenkunde zur Geschichte von Byzanz (3241453), 2 Bde. Wiesbaden 1982. [KW]

KIENAST, Dietmar: Römische Kaisertabelle. Grundzüge einer römischen Kaiserchronologie. Darmstadt ${ }^{2} 1996$.

Late Antiquity. A Guide to the postclassical World. Hrsg. v. Glen W. Bowersock / Peter Brown / Oleg Grabar. Cambridge, Mass.-London 1999.

Lexikon der Alten Welt. Zürich - Stuttgart 1965. [LAW]

Lexikon fir Theologie und Kirche. Freiburg i.Br. ${ }^{2} 1957-21968 ;{ }^{3} 1993-{ }^{32} 2001$. [LThK]

LidDELL, Henry G. / SCOTT, Robert: A Greek-English Lexicon. With a revised supplement. Oxford 1996. 